УДК 517.53

M. M. SHEREMETA

\title{
ON THE $l$-INDEX BOUNDEDNESS OF SOME COMPOSITION OF FUNCTIONS
}

\begin{abstract}
M. M. Sheremeta. On the l-index boundedness of some composition of functions, Mat. Stud. 47 (2017), 207-210.

It is suggested that for an entire function $f$ the function $F(z)=f\left(\frac{q}{(1-z)^{n}}\right), n \in \mathbb{N}$, is of bounded $l$-index with $l(|z|)=\frac{\beta}{(1-|z|)^{n+1}}, \beta>1$, if and only if $f$ is of bounded index.
\end{abstract}

1. Introduction. Let $0<R \leq+\infty, \mathbb{D}_{R}=\{z:|z|<R\}$ and $l$ be a positive continuous function on $[0, R)$, which satisfies

$$
l(r)>\frac{\beta}{R-r}, \quad \beta=\text { const }>1 .
$$

An analytic in $\mathbb{D}_{R}$ function $f$ is said $([1$, p. 67$])$ to be of bounded $l$-index if there exists $N \in \mathbb{Z}_{+}$such that for all $n \in \mathbb{Z}_{+}$and $z \in \mathbb{D}_{R}$

$$
\frac{\left|f^{(n)}(z)\right|}{n ! l^{n}(|z|)} \leq \max \left\{\frac{\left|f^{(k)}(z)\right|}{k ! l^{k}(|z|)}: 0 \leq k \leq N\right\} .
$$

The least such integer is called the $l$-index of $f$ and is denoted by $N(l ; f)$. If $R=+\infty$ (i. e. $f$ is an entire function) then the condition (1) is unnecessary. We remark also that if $f$ is an entire function and $l(|z|) \equiv 1$ then $f$ is said to be of bounded index.

A series of works is dedicated to the research of the $l$-index boundedness for different classes of analytic functions. For example, the $l$-index boundedness of entire functions represented by canonical products and Laguerre-Pólya functions is investigated in the papers [2-7]. The same problem is studied for analytic in the unit disc functions represented by Blaschke and Naftalevich-Tsuji products in [8-10].

In [11] it is proved that if $f$ is an entire function and $F(z)=f\left(q z^{n}\right) n \geq 2$, then the function $F$ is of bounded $l$-index with $l(|z|)=|z|^{n-1}$ for $|z| \geq 1$ if and only if $f$ is of bounded index. The following question arises: whether it is possible in this statement replace $q z^{n}$ by $\frac{q}{(1-z)^{n}}$ and $l(|z|)=|z|^{n-1}$ by $l(|z|)=\frac{\beta}{(1-|z|)^{n+1}}, \beta>1$. Here we give some elementary functions for which such a replacement is possible.

We need some notations. Suppose that $f$ is an analytic in $\mathbb{D}=\mathbb{D}_{1}$ function and $l(|z|)=$ $L\left(\frac{1}{1-|z|}\right), L(x) / x>\beta>1$ for $x \geq 1$. Then (2) is equivalent to

$$
\frac{\left|f^{(n)}(z)\right|}{n ! L^{n}(1 /(1-|z|))} \leq \max \left\{\frac{\left|f^{(k)}(z)\right|}{k ! L^{k}(1 /(1-|z|))}: 0 \leq k \leq N\right\} .
$$

2010 Mathematics Subject Classification: 30B50.

Keywords: analytic function; index boundedness; composition of functions.

doi:10.15330/ms.47.2.207-210

(C) M. M. Sheremeta, 2017 
For $r \in[0, \beta)$ we define

$$
\begin{aligned}
& \lambda_{1}(r)=\inf \left\{\frac{1}{L(x)} L\left(\frac{x}{1+t x / L(x)}\right):-r \leq t \leq r, x \geq 1\right\}, \\
& \lambda_{2}(r)=\sup \left\{\frac{1}{L(x)} L\left(\frac{x}{1+t x / L(x)}\right):-r \leq t \leq r, x \geq 1\right\} .
\end{aligned}
$$

By $Q_{\beta}$ we denote the class of the continuous in $[0, \beta)$ functions $L$ such that $L(x) / x>\beta>1$ for $x \geq 1$ and $0<\lambda_{1}(r) \leq \lambda_{2}(r)<+\infty$ for all $r \in[0, \beta)$. Then [12] (see also [1, p. 21]) the following statement is true.

Lemma 1. If $\beta>1$ and $L \in Q_{\beta}$ then (3) holds if and only if there exist numbers $p \in \mathbb{Z}_{+}$ and $C>0$ such that for each $z \in \mathbb{D}$

$$
\frac{\left|f^{(p+1)}(z)\right|}{L^{p+1}(1 /(1-|z|))} \leq C \max \left\{\frac{\left|f^{(k)}(z)\right|}{L^{k}(1 /(1-|z|))}: 0 \leq k \leq p\right\} .
$$

The function $L(x)=\beta x^{n+1}$ belongs to $Q_{\beta}$. Therefore, Lemma 1 implies the following lemma.

Lemma 2. If $l(|z|)=\frac{\beta}{(1-|z|)^{n+1}}, \beta>1$, then an analytic function $f$ in $\mathbb{D}=\mathbb{D}_{1}$ is of bounded $l$-index if and only if there exist numbers $p \in \mathbb{Z}_{+}$and $C>0$ such that for each $z \in \mathbb{D}$

$$
\frac{\left|f^{(p+1)}(z)\right|}{l^{p+1}(|z|)} \leq C \max \left\{\frac{\left|f^{(k)}(z)\right|}{l^{k}(|z|)}: 0 \leq k \leq p\right\}
$$

If $f(\xi)=e^{\xi}$ then $F(z)=\exp \left\{\frac{q}{(1-z)^{n}}\right\}, F^{\prime}(z)=\exp \left\{\frac{q}{(1-z)^{n}}\right\} \frac{q n}{(1-z)^{n+1}}$ and

$$
\begin{gathered}
F^{\prime \prime}(z)=\exp \left\{\frac{q}{(1-z)^{n}}\right\} \frac{q^{2} n^{2}}{(1-z)^{2 n+2}}+\exp \left\{\frac{q}{(1-z)^{n}}\right\} \frac{q n(n+1)}{(1-z)^{n+2}}= \\
=\frac{n+1}{1-z} F^{\prime}(z)+\frac{q^{2} n^{2}}{(1-z)^{2 n+2}} F(z),
\end{gathered}
$$

whence

$$
\begin{gathered}
\frac{\left|F^{\prime \prime}(z)\right|}{l^{2}(|z|)} \leq \frac{n+1}{(1-|z|) l(|z|)} \frac{\left|F^{\prime}(z)\right|}{l(|z|)}+\frac{|q|^{2} n^{2}}{(1-|z|)^{2 n+2} l^{2}(|z|)}|F(z)| \leq \\
\leq \frac{n+1}{\beta} \frac{\left|F^{\prime}(z)\right|}{l(|z|)}+\frac{|q|^{2} n^{2}}{\beta^{2}}|F(z)| \leq\left(\frac{n+1}{\beta}+\frac{|q|^{2} n^{2}}{\beta^{2}}\right) \max \left\{\frac{\left|F^{\prime}(z)\right|}{l(|z|)},|F(z)|\right\}
\end{gathered}
$$

that is (4) holds with $p=2$ and $C=\frac{n+1}{\beta}+\frac{|q|^{2} n^{2}}{\beta^{2}}$ and by Lemma 2 the function $F(z)=$ $\exp \left\{\frac{q}{(1-z)^{n}}\right\}$ is of bounded $l$-index with $l(|z|)=\frac{\beta}{(1-|z|)^{n+1}}, \beta>1$.

It is easy to show that for the functions $f(\xi)=\operatorname{ch} \xi$ and $f(\xi)=\operatorname{sh} \xi$ the equality

$$
F^{\prime \prime}(z)=\frac{n+1}{1-z} F^{\prime}(z)+\frac{q^{2} n^{2}}{(1-z)^{2 n+2}} F(z),
$$

is correct and for the functions $f(\xi)=\cos \xi$ and $f(\xi)=\sin \xi$ we have

$$
F^{\prime \prime}(z)=\frac{n+1}{1-z} F^{\prime}(z)-\frac{q^{2} n^{2}}{(1-z)^{2 n+2}} F(z) .
$$


Therefore, as above we get that the functions $F(z)=\operatorname{ch}\left\{\frac{q}{(1-z)^{n}}\right\}, F(z)=\operatorname{sh}\left\{\frac{q}{(1-z)^{n}}\right\} F(z)=$ $\cos \left\{\frac{q}{(1-z)^{n}}\right\}$ and $F(z)=\sin \left\{\frac{q}{(1-z)^{n}}\right\}$ are of bounded $l$-index with $l(|z|)=\frac{\beta}{(1-|z|)^{n+1}}, \beta>1$.

We remark that the entire functions $e^{z}, \operatorname{ch} z, \operatorname{ch} z, \cos z$ and $\sin z$ are of bounded index and satisfy a differential equation of the form $w^{\prime \prime}+a w=0$. S.M. Shah ([13]) considered a more general differential equation

$$
z^{2} w^{\prime \prime}+\left(\beta_{0} z^{2}+\beta_{1} z\right) w^{\prime}+\left(\gamma_{0} z^{2}+\gamma_{1} z+\gamma_{2}\right) w=0
$$

where $\beta_{0}, \beta_{1}, \gamma_{0}, \gamma_{1}, \gamma_{2}$ are constant parameters, and investigated the close-to-convexity of its solutions.

Suppose that an entire function $f(\xi)$ satisfies (5), that is

$$
\xi^{2} f^{\prime \prime}(\xi)+\left(\beta_{0} \xi^{2}+\beta_{1} \xi\right) f^{\prime}(\xi)+\left(\gamma_{0} \xi^{2}+\gamma_{1} \xi+\gamma_{2}\right) f(\xi) \equiv 0
$$

Let $F(z)=f\left\{\frac{q}{(1-z)^{n}}\right\}$. Since

$f^{\prime}\left\{\frac{q}{(1-z)^{n}}\right\}=\frac{(1-z)^{n+1}}{q n} F^{\prime}(z), f^{\prime \prime}\left\{\frac{q}{(1-z)^{n}}\right\}=\frac{(1-z)^{2 n+2}}{q^{2} n^{2}} F^{\prime \prime}(z)-\frac{(n+1)(1-z)^{n+1}}{q^{2} n^{2}} F^{\prime}(z)$,

from (6) we have

$$
\begin{gathered}
\frac{q^{2}}{(1-z)^{2 n}}\left(\frac{(1-z)^{2 n+2}}{q^{2} n^{2}} F^{\prime \prime}(z)-\frac{(n+1)(1-z)^{n+1}}{q^{2} n^{2}} F^{\prime}(z)\right)+ \\
+\left(\beta_{0} \frac{q^{2}}{(1-z)^{2 n}}+\beta_{1} \frac{q}{(1-z)^{n}}\right) \frac{(1-z)^{n+1}}{q n} F^{\prime}(z)+ \\
\quad+\left(\gamma_{0} \frac{q^{2}}{(1-z)^{2 n}}+\gamma_{1} \frac{q}{(1-z)^{n}}+\gamma_{2}\right) F(z) \equiv 0,
\end{gathered}
$$

that is

$$
\begin{gathered}
F^{\prime \prime}(z)+\left(\frac{\beta_{0} n q-(n+1)}{(1-z)^{n+1}}+\frac{\beta_{1} n}{(1-z)}\right) F^{\prime}(z)+ \\
+n^{2}\left(\gamma_{0} \frac{q^{2}}{(1-z)^{2 n+2}}+\gamma_{1} \frac{q}{(1-z)^{n+2}}+\frac{\gamma_{2}}{(1-z)^{2}}\right) F(z) \equiv 0 .
\end{gathered}
$$

If $l(|z|)=\frac{\beta}{(1-|z|)^{n+1}}, \beta>1$, hence we obtain

$$
\begin{aligned}
& \frac{\left|F^{\prime \prime}(z)\right|}{l^{2}(|z|)} \leq \frac{\left|n q \beta_{0}-n-1\right|+n\left|\beta_{1}\right|}{(1-|z|)^{n+1} l(|z|)} \frac{\left|F^{\prime}(z)\right|}{l(|z|)}+n^{2} \frac{\left|\gamma_{0} q^{2}\right|+\left|\gamma_{1} q\right|+\left|\gamma_{2}\right|}{(1-|z|)^{2 n+2} l^{2}(|z|)}|F(z)| \leq \\
\leq & \left(\frac{\left|n q \beta_{0}-n-1\right|+n\left|\beta_{1}\right|}{\beta}+\frac{n^{2}\left(\left|\gamma_{0} q^{2}\right|+\left|\gamma_{1} q\right|+\left|\gamma_{2}\right|\right)}{\beta^{2}}\right) \max \left\{\frac{\left|F^{\prime}(z)\right|}{l(|z|)},|F(z)|\right\}
\end{aligned}
$$

that is by Lemma $2 F$ is of bounded $l$-index. We remark also that from $(6)$ it follows that $f$ is of bounded index. Indeed, for $|\xi| \geq 1$

$$
\begin{gathered}
\left|f^{\prime \prime}(\xi)\right| \leq\left(\left|\beta_{0}\right|+\left|\beta_{1}\right|\right)\left|f^{\prime}(\xi)\right|+\left(\left|\gamma_{0}\right|+\left|\gamma_{1}\right|+\left|\gamma_{2}\right|\right)|f(\xi)| \leq \\
\quad \leq\left(\left|\beta_{0}\right|+\left|\beta_{1}\right|+\left|\gamma_{0}\right|+\left|\gamma_{1}\right|+\left|\gamma_{2}\right|\right) \max \left\{\left|f^{\prime}(\xi)\right|,|f(\xi)|\right\}
\end{gathered}
$$

that is by Hayman's theorem [14] (see also Theorem 1.5 from [1] with $l(|z|) \equiv 1$ ) $f$ is of bounded index in $\mathbb{C} \backslash \mathbb{D}$ and, thus [1, p. 32], $f$ is of bounded index.

In view of the results given above we can propound following conjecture. 
Conjecture 1. For an entire function $f$ the function $F(z)=f\left(\frac{q}{(1-z)^{n}}\right), n \in \mathbb{N}$, is of bounded l-index with $l(|z|)=\frac{\beta}{(1-|z|)^{n+1}}, \beta>1$, if and only if $f$ is of bounded index.

Finally, we remark that there is a number of works [15-17] devoted to entire solutions of the differential equation (5). Their main results are estimates of the index with additional conditions on the parameters.

\section{REFERENCES}

1. Sheremeta M.M. Analytic functions of bounded index. - Lviv: VNTL Publishes, 1999.

2. Goldberg A.A., Sheremeta M.N. Existence of an entire transcendental function of bounded l-index// Mat. Zametki. - 1995. - V.57, no.1 - P. 125-129 (in Russian); Engl. transl. Math Notes. - 1995. - V.57, no.1. - P. 88-90.

3. Sheremeta M.M., Bordulyak M.T. On the existence of entire functions of bounded l-index and l-regular growth// Ukrainian Math. Zh. - 1996. - V.48, no.9. - P. 1166-1182 (in Russian); Engl. transl.: Ukrainian Math. J. - 1996. - V.48, no.9. - P. 1322-1340.

4. Chyzhykov I.E., Sheremeta M.M. Boundedness of l-index for entire functions of zero genus// Mat. Stud. - 2001. - V.16, no.2. - P. 124-130.

5. Sheremeta M.M., Bordulyak M.T. Boundedness of the l-index of Laguerre-Pólya entire functions// Ukrainian Math. Zh. - 2003. - V.55, no.1. - P. 91-99 (in Ukrainian); Engl. transl.: Ukrainian Math. J. - 2003. - V.55, no.1, 112-125.

6. Chyzhykov I.E., Sheremeta M.M. On the boundedness of l-index for entire functions of zero genus// Dopovidi NAN Ukraine. - 2003. - V.7. - P. 33-39. (in Ukrainian)

7. Goldberg A.A., Sheremeta M.M. On the boundedness of the l-index of canonical products// Ukr. Mat. Visn. - 2005. - V.2, no.1. - P. 52-64 (in Ukrainian); Eng. transl.: Ukr. Math. Bull. - 2005. - V.2, no.1. - P. 53-65.

8. Trukhan Yu.S., Sheremeta M.M. Boundedness of l-index of Blaschke product// Mat. Stud. - 2002. V.17, no.2. - P. 127-137. (in Ukrainian)

9. Trukhan Yu.S., Sheremeta M.M. On l-index boundedness of the Blaschke product// Mat. Stud. - 2003. - V.19, no.1. - P. 106-112.

10. Sheremeta M.M., Trukhan Yu.S. Boundedness of the l-index of the Naftalevich-Tsuji product// Ukrainian Math. Zh. - 2004. - V.56, no.2. - P. 247-256 (in Ukrainian); Engl. transl.: Ukrainian Math. J. - 2004. - V.56, no.2. - P. 305-317.

11. Sheremeta M.N. Entire functions and Dirichlet series of bounded l-index// Izv. Vyssh. Uchebn. Zaved. Mat. - 1992. - V.9. - P. 81-87. (in Russian). Engl. transl.: Russian Math. (Iz. VUZ). - 1992. - V.36, no.9. - P. 76-82.

12. Strochyk S.M., Sheremeta M.M. Analytic in the unit disc functions of bounded index// Dopov. Akad. Nauk Ukr. - 1993. - no.1. - P. 19-22. (in Ukrainian)

13. Shah S.M. Univalence of a function $f$ and its successive derivative when $f$ satisfies a differential equation, II// J. Math. Anal. and Appl. - 1989. - V.142. - P. 422-430.

14. Hayman W.K. Differential inequalities and local valency// Pacific J. Math. - 1973. - V.44. - P. $117-137$.

15. Sheremeta Z.M. Properties of derivatives of an entire solution of a differential equation// Mat. method. and fiz.-mech. polya. - 2006. - V.49, no.2. - P. 80-85. (in Ukrainian)

16. Sheremeta Z.M., Sheremeta M.M. Properties of entire solutions of differential equations// Ukrainian Math. Zh. - 2006. - V.58, no.12. - P. 1693-1703 (in Ukrainian); Engl. transl.: Ukrainian Math. J. 2006. - V.56, no.12. - P. 1924-1934.

17. Sheremeta Z.M., Sheremeta M.M. On the l-index boundedness of entire solutions of a differential equation// Dopovidi NAN Ukraine. - 2007. - V.56, no.2. - P. 31-36. (in Ukrainian)

Ivan Franko National University of Lviv

m_m_sheremeta@gmail.com 